\title{
Mapas de ideas: una herramienta para el aprendizaje escolar. Datos y comentarios para una discusión
}

\author{
Eduardo Vidal-Abarca y Ramiro Gilabert
}

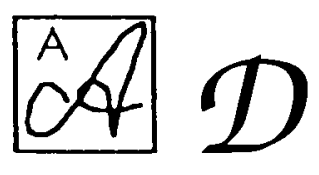

Desde los estudios que tratan de llevar la «espacialidad del pensamiento» al propio pensamiento y aprendizaje verbal, el «mapa de ideas» ha ido difundiéndose entre los educadores como una alternativa potente y fácil de usar para estructurar y representar los conocimientos en ese lenguaje mixto gráfico-verbal. Se describe aqui con detalle la técnica y un ejemplo concreto de aplicación.

Desde hace unos pocos años, y especialmente en países anglosajones, está cobrando importancia el uso de diversas técnicas que enseñan a los estudiantes a representar visualmente el contenido de los textos que tienen que estudiar, como un medio para mejorar el aprendizaje. Dichas técnicas posibilitan la transformación de la información textual desde un formato secuencial propio de los textos, a otro visual, más interconectado y global. El procedimiento general de todas ellas consiste en construir redes formadas por «expresiones-nudo" y «expresiones-conexión». Las primeras representan conceptos, acciones o ideas, mientras que los segundos indican la relación existente entre las primeras. Algunas de estas técnicas son las siguientes: «redes semánticas» (networking) propuestas por Dansereau y colaboradores (Holley \& Dansereau, 1984), "diagramas de flujo» (flowcharting) por Geva (1984), o la técnica denominada «mapa-de-ideas» (idea-mapping) por Armbruster y colaboradores (Armbruster \& Anderson, 1982; ver una exposición resumida en Vidal-Abarca y Gilabert, 1991, cap. 7). Nosotros aquí emplearemos el término mapas de ideas para referirnos a todas ellas, dado que nos parece el nombre más inclusivo.

El objetivo de este trabajo es exponer los fundamentos psicológicos que subyacen a estas técnicas, así como una muestra de su utilización como herramientas para mejorar las destrezas de comprensión y aprendizaje de los alumnos. De esta forma, aquellos profesionales que decidan conocerlas más a fondo y, en su caso, utilizarlas tendrán más elementos de juicio para evaluar su eficacia y tomar decisiones sobre su aplicación. Así, comenzaremos exponiendo brevemente las razones teóricas que subyacen al surgimiento de las técnicas de mapas de ideas. A continuación expondremos de forma más detallada una de ellas, señalando los problemas que tiene su aplicación. Posteriormente mencionaremos algunos datos sobre su utilización procedentes de una investigación realizada por nosotros mismos. Finalmente, haremos unos comentarios que sinteticen nuestras conclusiones y que puedan servir para una discusión con todos los profesionales interesados en ello. 


\section{Los mapas de ideas: unas notas sobre su fundamento psicológico}

Las técnicas de «mapas de ideas» se fundamentan en dos tipos de estudios sobre la organización del conocimiento los cuales tienen su origen en investigaciones realizadas por psicólogos cognitivos en los años 70 y 80 , a saber: estudios sobre redes proposicionales y estudios sobre esquemas. En las líneas siguientes haremos un breve resumen de ambos estudios.

Las personas poseemos múltiples conocimientos sobre los más variados temas. Pero, ¿cómo se puede representar la organización de esos conocimientos en la memoria humana? Los psicólogos cognitivos han intentado elaborar y verificar modelos teóricos sobre este tema. Cuando se investiga sobre la memoria humana, hay un hecho experimental ampliamente comprobado: las personas recordamos el significado de acontecimientos o afirmaciones, pero no recordamos tan fielmente las expresiones literales. Así, la frase «Bofill Jr. regaló un anillo millonario a Chabeli, la hija de Julio Iglesias» es díficilmente distinguible de esta otra: «La hija de Julio Iglesias, Chabeli, recibió de Bofill Jr. como regalo un anillo millonario». La razón de que sean difícil distinguir ambas frases es que las dos trasmiten estas tres ideas: 1. «Bofill Jr. regaló un anillo a Chabeli», 2. "el anillo era millonario", 3. "Chabeli es la hija de Julio Iglesias». Es decir, las personas tendemos a captar ideas más que expresiones literales. Estas ideas simples se denominan "proposiciones" y pueden ser representadas en forma lineal o en forma visual. Esta última forma se puede ver en la Figura 1.

FigurA 1

Red proposicional para representar la frase:

«Bofill Jr. regaló un anillo millonario a Chabeli, la bija de Julio Iglesias»

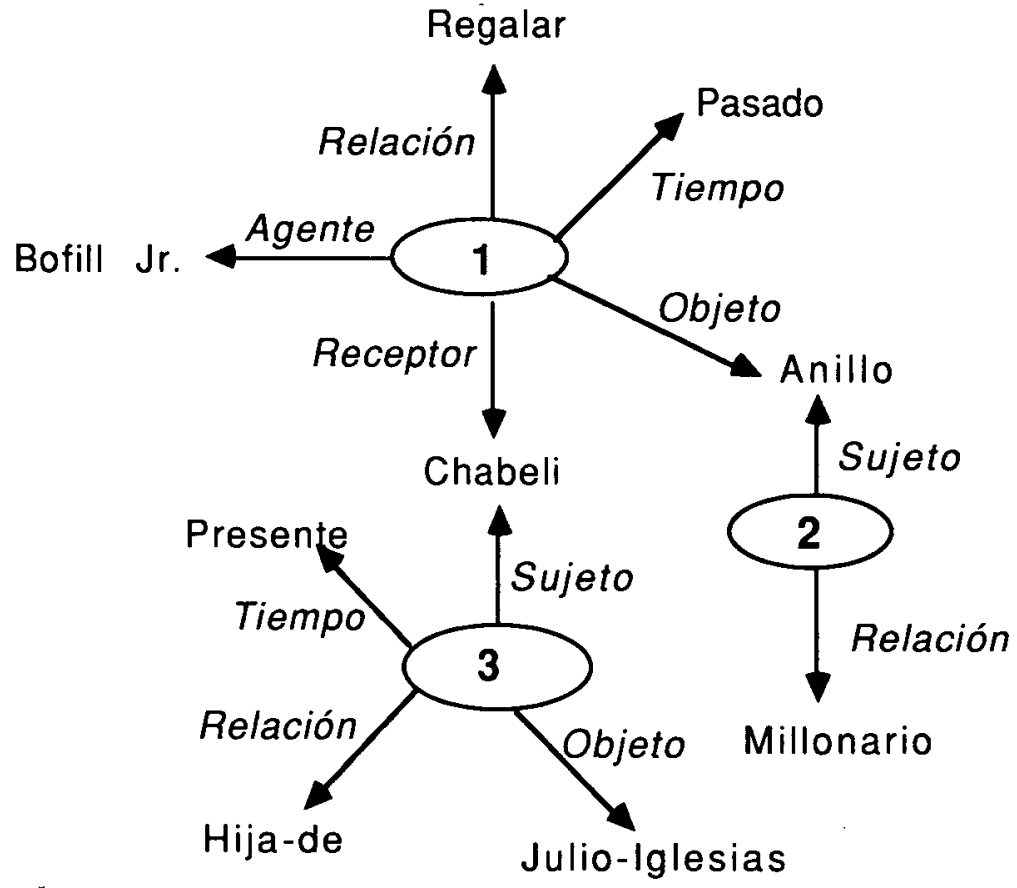

Las representaciones visuales tienen la ventaja de que permiten representar las interconexiones entre ideas. Estas representaciones visuales se denominan «redes proposicionales» y constan de «nudos» y «conexiones». Los «nudos» pueden ser de tres tipos: proposiciones, relaciones y argumentos. Las «proposiciones» son las ideas que antes citamos (p.e. «Bofill Jr. regaló un anillo a Chabeli»). Se representan por 
números encerrados en un óvalo (ver la figura 1). En la figura se aprecian tres proposiciones que se corresponden con las tres ideas antes mencionadas. Los «argumentos» son nombres (p.e. «Bofill Jr.», "Chabeli», «anillo», etc). Las «relaciones» son términos que unen argumentos tales como verbos (p.e. "regalar»), u otras expresiones (p.e. «hija-de»), o bien términos que califican argumentos, como los adjetivos (p.e. «millonario»). Tanto los «argumentos» como las «relaciones» forman parte de las proposiciones. Los nudos están unidos por conexiones, las cuales son un número finito (en la figura 1 se pueden ver algunos ejemplos de conexiones escritas en cursiva, tales como agente, objeto, sujeto, tiempo, etc).

Así, el óvalo 3 representa la proposición 3 («Chabeli es la hija de Julio Iglesias»). «Chabeli» y «Julio-Iglesias» son argumentos, e «hija-de» es la relación que une a ambos argumentos. A su vez, las conexiones que unen a esos términos dentro de la proposición son, sujeto en el caso de Chabeli, objeto en el caso de Julio-Iglesias. Ambos están conectados por la relación «hija-de» en un tiempo presente (indicado por el término «es» hija-de, pero no «fue» o «será» hija-de).

¿Por qué representar el conocimiento de las personas mediante redes proposicionales? Porque estos modelos son congruentes con diversos hechos empíricos sobre cómo las personas reconocemos, recordamos y recuperamos la información almacenada en nuestra memoria. Es decir, las redes proposicionales son un modelo adecuado para representar cómo el conocimiento está estructurado en la memoria.

Los estudios sobre esquemas de conocimiento son otro fuerte apoyo teórico para las técnicas de mapas de ideas. Los esquemas de conocimiento son formas de representar las regularidades que presentan distintos objetos, acciones o ideas. Así, por ejemplo, todos tenemos una esquema de lo que es una "casa». El mismo se concreta en una serie de atributos (p.e. materiales, función, forma, componentes) y una serie de variables que rellenan esos atributos (p.e. una casa puede estar hecha por diversos materiales tales como: ladrillo, madera, adobe, etc). Si comparamos las proposiciones con los esquemas vemos que las primeras representan lo que es importante sobre cosas o acontecimientos, mientras los esquemas representan aquello que las cosas específicas tienen en común. Los esquemas tienen la propiedad de estar incluidos unos en otros (p.e. «casa» está incluido en «edificio»), de forma que el esquema incluido ( «casa») hereda las características del más amplio («edificio»). Estas características se infieren sin necesidad de hacerlas explícitas. Ello facilita el recuerdo y la comprensión, ya que un esquema se puede relacionar con otros a los que incluye o en los que está incluido. Además, permite organizar y jerarquizar el conocimiento, conectando unos esquemas con otros. Es decir, esta teoría afirma que el conocimiento humano está «organizado» y «jerarquizado» en esquemas y subesquemas que heredan las propiedades de aquellos otros que tienen mayor nivel de generalidad.

Los psicólogos han estudiado diversos tipos de esquemas. Entre otros podemos citar los siguientes: esquemas referidos a objetos (p.e. «casa»), a acciones (p.e. «ir a un restaurante»), a conceptos (p.e. «ser vivo»), a tipos de textos (p.e. «narración infantil o cuento», «descripción de un fenómeno», o explicación de las «causas/efectos" de fenómenos", etc). Los esquemas de conceptos y los de tipos de textos son los más interesantes para las técnicas de mapas de ideas. Diversos hallazgos experimentales avalan este interés. Así, cuando se analiza el papel de los esquemas en la comprensión y el recuerdo de información textual, se encuentra que, al estudiar un texto, los buenos lectores:

1. Seleccionan la información importante y jerarquizan las ideas del texto y conectan unas ideas con otras basándose en los esquemas que poseen.

2. Acomodan la información que reciben a sus esquemas. Así, pueden inferir información que no estaba presente en el texto, reordenar la información para acomodarla al esquema previo, etc. 
3. Reconocen el esquema o esquemas que están presenten en el texto y lo emplean para recuperar la información que han leído.

Resumiendo, las aportaciones de los estudios sobre organización del conocimiento a las técnicas de mapas de ideas se pueden concretar en las siguientes:

1. Las personas poseemos ideas sobre objetos y acontecimientos que se pueden representar en forma de «proposiciones».

2. La relación entre proposiciones se puede representar en forma de redes proposicionales, las cuales tienen un formato visual-espacial.

3. Las redes proposicionales constan de «nudos» (potencialmente infinitos) y «conexiones» (en número finito)

4. Las regularidades entre objetos, conceptos, ideas o acontecimientos se pueden representar en forma de esquemas. jerárquica.

5. Los esquemas permiten representar la información de forma organizada y

Aparte del fundamento psicológico que acabamos de mencionar, las técnicas de mapas de ideas guardan semejanza con los estudios sobre «mapas conceptuales» (Novak y Gowin, 1988). Estos estudios provienen de una tradición diferente a la mencionada previamente. Su interés es fundamentalmente aplicado: cómo representar el conocimiento que un sujeto tiene de un tema educativo concreto. Además, su fundamento teórico es la teoría del aprendizaje significativo de D.P. Ausubel, una teoría cognitiva diferente del enfoque psicológico del «procesamiento de la información» que es el marco en que hay que entender los estudios psicológicos sobre representación del conocimiento mencionados anteriormente. Por último, hay que señalar que los mapas conceptuales se centran más en representar conceptos y sus relaciones entre ellos, mientras que las técnicas de mapas de ideas representan preferentemente ideas (proposiciones) y relaciones entre ellas. Por lo demás, hay que decir que ambas técnicas tienen semejanzas muy considerables. De hecho, y por lo que respecta a las aplicaciones didácticas, no hay diferencias sustanciales entre la técnica de «mapas de ideas» y la de «mapas conceptuales» (ver, por ejemplo, el trabajo de Fernández, 1992, sobre la utilización de mapas conceptuales en la enseñanza de la historia).

\section{Breve revisión de la técnica de mapas de ideas}

Las técnicas de mapas de ideas enseñan a los estudiantes a seleccionar la información importante de un texto, a conectar unas ideas con otras, a organizarlas en forma jerárquica y a representar el producto resultante en un formato visual-espacial. Todas estas operaciones mentales forman parte esencial de la comprensión. Es decir, para comprender es preciso distinguir lo esencial de lo accesorio, encontrar relaciones entre las ideas del texto, especialmente entre las más importantes, y finalmente dar una organización jerárquica a las ideas y relaciones encontradas. Por tanto, elaborar un buen mapa de ideas supone implicarse en las actividades mentales que conducen a una comprensión profunda de la información que el texto pretende comunicar. Este es el valor principal y el sentido que tiene la utilización de la técnica de mapa de ideas.

Pero, ¿cómo se lleva a cabo todo eso? Ceñiremos nuestra exposición a la técnica mapa-de-ideas propuesta por Armbruster y colaboradores (Armbruster y Anderson, 1982) ya que es una de las más elaboradas. La técnica consiste en enseñar las relaciones y esquemas textuales básicos que se pueden encontrar en los textos que se estudian. Esto obliga a partir de un esquema previo de relaciones o conexiones posibles. $\mathrm{La}$ Figura 2 ilustra algunas de las relaciones posibles. Así, a los estudiantes se les explica que los conceptos e ideas del texto pueden estar conectados por un conjunto limitado de relaciones posibles, y se les enseña a identificarlas en el texto (p.e. «A es un ejemplo de $B$ », «A es una propiedad de $B$ », «A ocurre antes que $B$ », etc). 
FIGURA 2

Ejemplo de algunas de las relaciones entre ideas mencionadas por A rmbruster y Anderson (1982) dentro de la técnica llamada "mapa-de-ideas" (idea-mapping)

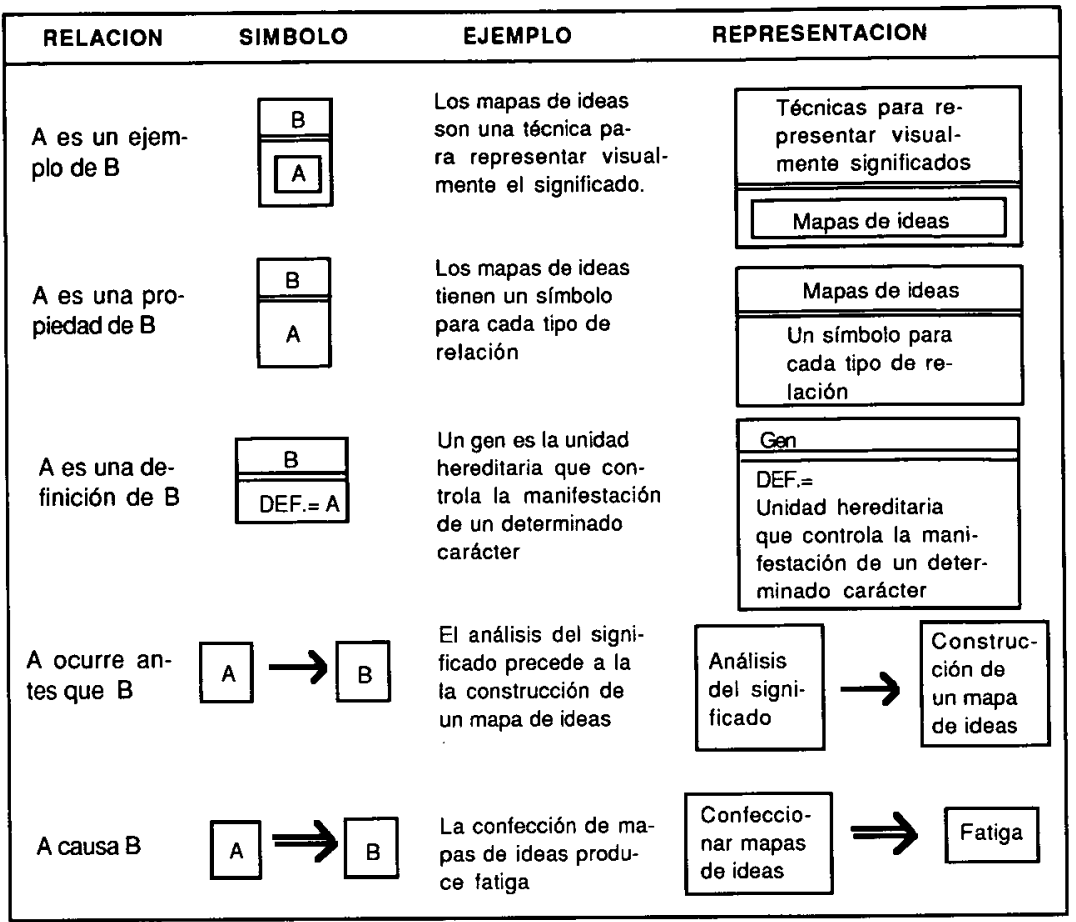

Además, se parte de la base de que existe un número limitado de esquemas para organizar los textos. Así, por ejemplo, Armbruster y colaboradores asumen que los textos que estudian los muchachos pueden tener los siguientes esquemas globales de organización: descripción, comparación/contraste, secuencia temporal, explicación/ causación y definición/ejemplo. El siguiente texto tiene una organización global de "secuencia temporal» $\mathrm{e}$ incluye relaciones de «secuencia» (A ocurre antes que $\mathrm{B}$ ) y de «causalidad» (A causa B). La representación del mismo en forma de mapa-de ideas según la técnica de estos autores se puede ver en la Figura 3.

Durante el día, cuando la temperatura es superior al punto de congelación del agua $\left(0^{\circ} \mathrm{C}\right)$, el agua de la lluvia, la nieve o el hielo derretidos se introducen entre las grietas de las rocas. Durante la noche, cuando la temperatura baja del punto de congelación del agua, el agua atrapada incrementa su volumen al convertirse en hielo.

Cuando este agua congelada aumenta de volumen, el hielo empuja las paredes de las grietas con tremenda fuerza, rompiendo la roca en trozos. De esta forma, grandes masas de rocas se rompen en trozos pequeños.

Los chicos van aprendiendo a representar tanto las relaciones entre ideas como los diferentes tipos de organización de la información de los textos combinando la presentación de ejemplos con la activación de esquemas previos. Así, se puede enseñar a los alumnos el texto anterior y el mapa de la Figura 3 incompleto. Tras su lectura, se les puede pedir que lo completen, enseñándoles las relaciones, los símbolos y las estructuras textuales de forma progresiva. En otros casos, se les puede presentar el título de un tema (p.e. «el aparato respiratorio») y solicitar que activen sus conocimientos previos sobre posibles contenidos de dicho tema (p.e. «función», «parte», «secuencia del proceso de la respiración», etc). 
Representación de un texto en forma de mapa-de-ideas siguiendo la técnica propuesta por Armbruster y Anderson (1982)
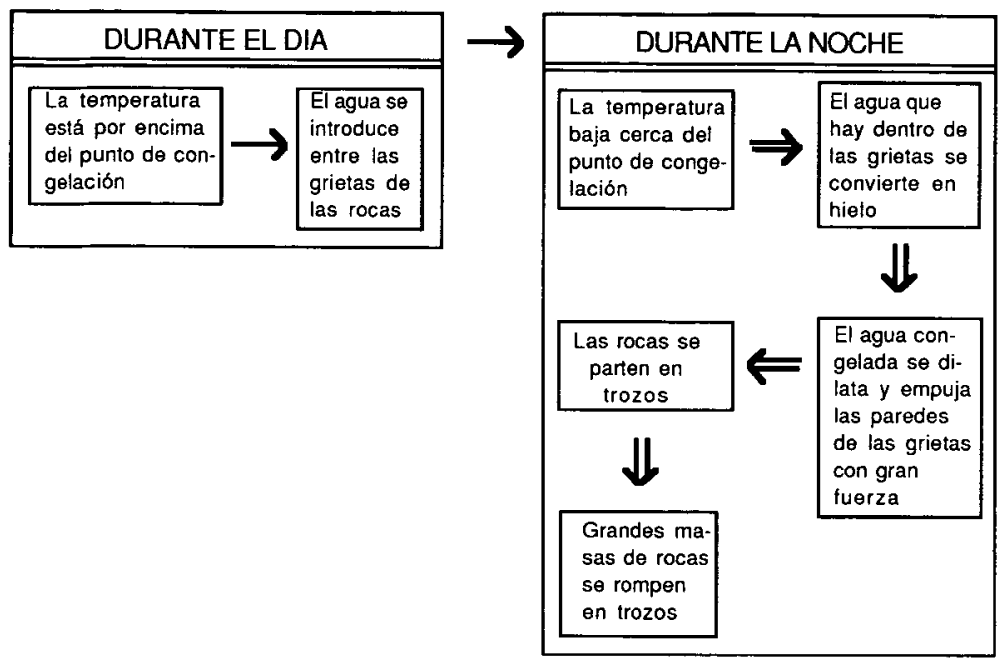

No obstante, la utilización de estas técnicas tiene dos problemas. El primero es que se require que los textos estén especialmente elaborados para adaptarse a los presupuestos antes citados. Es decir, tanto la organización de los textos como las relaciones entre las ideas se han de ajustar fielmente a los esquemas previamente definidos («secuencia temporal», «explicación/causación», etc; «A ocurre antes que B», «A causa $B "$, etc). Sin embargo, los textos ordinarios que estudian los alumnos no se ajustan a esos presupuestos. Así, suelen ser poco coherentes, no explicitan las relaciones entre ideas y con frecuencia carecen de una estructura definida (Sánchez, 1993). Es decir, es muy complicado, e incluso en ocasiones imposible, conseguir que los estudiantes elaboren un buen mapa de ideas a partir de un tema sacado de un libro de texto.

El segundo problema es importante sobre todo cuando se utiliza la técnica de mapa de ideas con chicos menores de 14-15 años. Estos alumnos tienen muchas dificultades para pensar en términos formales y abstractos, lo cual es fundamental cuando se trata de comprender estructuras textuales y relaciones abstractas entre ideas. Es decir, a los chicos de 10-14 años les cuesta enormemente tanto codificar relaciones entre ideas en la forma mencionada ( $A$ A ocurre antes que $B » ;$; $A$ causa $B » ;$ « $A$ es una definición de $B$ »; «A es un ejemplo de $B$ », etc), como reconocer estructuras textuales (secuencia temporal, explicación/causación, etc). Este problema está estrechamente ligado al anterior, ya que la tarea de codificar relaciones o reconocer estructuras textuales es mucho más complicada cuando ambas no están explícitas en el texto, que cuando sí lo están. Dado que lo primero es lo más frecuente, la tarea resulta especialmente difícil para estos estudiantes.

Una solución a los dos problemas mencionados consiste en aplicar procedimientos que faciliten la transición desde las condiciones instruccionales hasta las condiciones académicas reales. Así, pensamos que es positivo comenzar la instrucción con textos adaptados en los cuales las relaciones entre ideas y las estructuras textuales sean más claras. De esta forma los sujetos de niveles escolares medios serán capaces de entender ambos elementos textuales más fácilmente que cuando estudian textos de materiales académicos ordinarios. A continuación se deberían emplear diversos procedimientos para pasar de los textos adaptados a los ordinarios. Estos procedimientos podrían implicar un análisis de aquellas características textuales que diferencian ambos tipos de textos, especialmente de las que parezcan más adecuadas 
teniendo en cuenta las demandas de la tarea y las posibilidades congnitivas de los sujetos. Por lo que respecta a la dificultad de comprender relaciones abstractas entre ideas y estructuras textuales básicas, el profesor puede tener presente un conjunto posible de ellas, no tanto para enseñarlas expresamente cuanto para tener un marco general flexible de posibles relaciones y estructuras (ver Sánchez, 1993 para una excelente exposición de las estructuras textuales y de su utilidad en la enseñanza de estrategias de comprensión).

\section{Nuestro sistema de enseñanza de mapas de ideas: algunos datos}

Con estos presupuestos, llevamos a cabo un programa para enseñar a niños de $6^{\circ}$ curso cómo representar el contenido de las lecciones de Ciencias Sociales y Ciencias Naturales en forma de mapa de ideas. El mismo fue llevado a cabo por los profesores de esas áreas en dos aulas de un colegio público de una población cercana a Valencia. La exposición pormenorizada de la metodología y resultados de este trabajo se pueden ver en Vidal-Abarca, Gilabert y Garcia Madruga (1993). Aquí nos centraremos en la explicación del programa, haciendo un breve resumen de los resultados.

El programa de instrucción que siguieron los niños estuvo dividido en tres fases. Dedicamos once sesiones de una hora aproximada de duración a la primera fase en la cual se enseñó a los chicos a representar el contenido de los textos en forma de mapa de ideas. Para ello, se adaptaron los textos de seis lecciones del libro de Ciencias Naturales y de otras cinco del de Ciencias Sociales. La adaptación se efectuó siguiendo diversas recomendaciones (muchas de ellas se especifican y comentan en Vidal-Abarca y Gilabert, 1991, y en Sánchez, 1993), consistiendo básicamente en incrementar la coherencia textual de forma que los estudiantes pudieran construir más fácilmente el mapa de ideas de cada lección. En la primera sesión, tras explicárseles el propósito general del programa, leyeron la versión adaptada del texto correspondiente. Posteriormente, extrajeron el tema y las ideas principales de cada párrafo o grupo de párrafos, haciendo luego otro tanto con todo el texto en su conjunto. A continuación se les enseñó cómo representar cada frase de un párrafo determinado, y todo el conjunto del mismo en forma de mapa de ideas. Finalmente, se les mostró el mapa de ideas de una lección completa, el cual tenía algunos huecos. Pedimos a los chicos que sugirieran respuestas para rellenar los huecos, comentando las diferentes soluciones y razonando la mayor o menor bondad de las mismas. Durante las siguientes sesiones de esta fase, el programa incluyó las siguientes actividades: lectura del texto de la lección por partes, decir el tema y la idea o ideas principales de cada una de las partes, hacer otro tanto con el conjunto del texto y, finalmente, completar un mapa de ideas del texto incluyendo sólo las ideas principales. En la figura 4 se puede ver uno de los mapas de ideas utilizados, si bien en este caso el mapa está sin huecos para dar una idea más completa al lector de las relaciones representadas. Conforme el programa fue avanzando se fueron suprimiendo ayudas a los estudiantes.

A lo largo de todo el proceso hicimos especial hincapié en las actividades encaminadas a relacionar y jerarquizar las ideas textuales con la finalidad de producir una representación integrada del contenido semántico del texto. Para ello, teníamos presente las posibles relaciones entre ideas que las distintas técnicas instruccionales referidas en la introducción proponían, aunque no hicimos un uso explícito de las mismas. Como se puede observar en el ejemplo citado, tampoco empleamos símbolos especiales para representar las relaciones entre ideas. Por el contrario, los nexos eran expresiones idénticas o sinónimas a las que estaban escritas en el texto original. Las ideas más generales se colocaban en la parte superior del mapa, tomando la dimensión vertical (arriba-abajo) como un indicador de la importancia de las ideas. Los distintos mapas de ideas de los chicos fueron ampliamente comentados durante esta fase de instrucción. Así mismo, insistimos en que lo importante eran las ideas que se expresaban, no las palabras empleadas. 
Figura 4

Ejemplo de mapa de ideas empleado en una de las sesiones del programa de instrucción

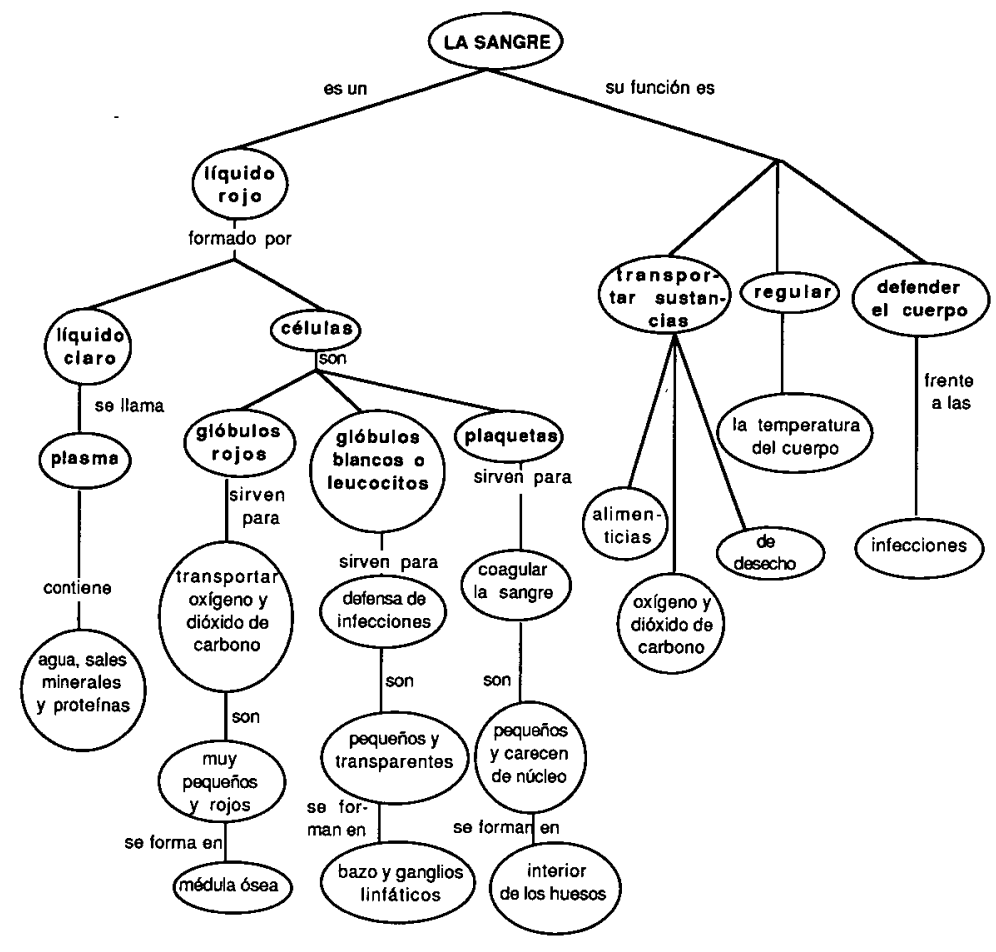

Durante las seis sesiones que duró la segunda fase, se enseñó a los muchachos a ser conscientes de algunas características de los textos ordinarios que podían interferir con su comprensión. Así, prestamos atención especial a tres características negativas de muchos de estos textos: la ausencia de expresiones que mencionen explícitamente la conexión entre ideas, la inadecuación entre los encabezamientos de algunas secciones del texto y los contenidos de las mismas, y la falta de coherencia global del texto. De esta forma intentamos facilitar la transición desde la lectura de textos adaptados hacia la de los textos ordinarios. Se presentaba a los niños dos versiones del mismo texto: la original del libro de texto y otra adaptada, siendo esta última más adecuada debido a los cambios efectuados en las características que acabamos de mencionar. Tras la lectura de ambas versiones se pedía a los chicos que indicaran sus diferencias y se comentaba el efecto que éstas podían tener a la hora de construir un mapa de ideas a partir de ellos. La tercera fase duró ocho sesiones. Los chicos practicaron la construcción de mapas de ideas con sus textos ordinarios de Ciencias Naturales y Sociales.

Los profesores ordinarios de las dos áreas citadas enseñaron a sus alumnos durante el período ordinario de clase. Previamente al período de instrucción hubo varias reuniones en las que lo autores de este trabajo explicamos brevemente a los profesores los fundamentos teóricos y las actividades del programa, además de procederse a la elaboración de gran parte de los materiales. A lo largo de la aplicación del programa fueron frecuentes las reuniones para comentar las observaciones de los profesores sobre dicho proceso de aplicación. Se empleó una metodología de instrucción directa si bien prestamos atención especial a la discusión y participación de los estudiantes.

¿Qué efectos encontramos tras la aplicación del programa? Hay datos cuantitativos referidos a una prueba de recuerdo y a otra de comprensión que se aplicaron a 
los niños antes y después comenzar las sesiones de enseñanza. Estos datos se compararon con los obtenidos por niños de otras dos aulas a los que no se les enseñó ningún procedimiento especial. Encontramos que los primeros mejoraron significativamente más que los segundos en la prueba de recuerdo; es decir, recordaban más ideas de un texto, especialmente las ideas más importantes. También mejoraron en las respuestas que daban a preguntas dirigidas a relacionar unas ideas con otras. Sin embargo no mejoraron en respuestas a preguntas en las que se pedía a los chicos que aplicaran la información del texto a situaciones nuevas. En la figura 5 se puede ver uno de los textos empleados para evaluar los efectos del programa, así como dos ejemplos de las preguntas de comprensión que les planteamos. Como se puede ver, para contestar a las primeras preguntas se requiere haber comprendido el texto. Sin embargo, las preguntas de aplicación requiren, además de comprender la información del texto, trasladar dicha información a una nueva situación análoga. Esta transferencia de información supone un nivel de aprendizaje más profundo.

FIGURA 5

Texto empleado para evaluar los efectos del programa y ejemplos de las cuestiones de comprensión plantedas a lor bbimi

TEXTO.

Los superpetroleros son los barcos que se dedican a transportar el petróleo a través del océano. Un superpetrolero de tipo medio tiene una capacidad de carga enorme y su tamaño es gigantesco. En sus bodegas podría caber un edificio de 100 pisos. Sin embargo, los superpetroleros causan graves problemas a la naturaleza que es necesario resolver; los superpetroleros, con frecuencia vierten el petróleo de su carga en los mares y por ello la vida del mar y de las costas sufre daños muy graves.

En 1967, un superpetrolero, el Torre Canyon, se partió en dos frente a las costas de Inglaterra, el petróleo derramado causó la muerte a 200.000 peces. En el año 1970, cerca de España, otro superpetrolero sufrió una explosión y el buque estalló en llamas; los restos del petróleo se mezclaron con la niebla, y dias más tarde se precipitó sobre las costas cercanas una lluvia negra que destruyó la cosecha. Además, el petróleo vertido en las aguas del océano destruye la vida de las plantas marinas que son muy importantes para la vida de la Tierra, ya que producen el setenta por ciento del oxígeno necesario.

La solución de estos problemas no es prohibir el uso de los superpetroleros. Los superpetroleros transportan la mayor parte del petróleo que consumimos y no existe otra forma de transportarlo. La solución, por el contrario, debe buscarse a través de estas medidas. En primer lugar, es necesario construir mejores superpetroleros, con mayor fuerza y resistencia. En segundo lugar, los oficiales de estos buques deberian ser entrenados de forma especial para poder manejarlos en situaciones de emergencia como son las tormentas. La tercera medida es instalar estaciones de control en los lugares por donde los superpetroleros se aproximan a las costas. Estas estaciones de control podrían actuar de forma semejante a las torres de control que se usan para los aviones; es decir, las estaciones de control podrían guiar a los superpetrolero en sus movimientos de aproximación a las costas y puertos.

\section{EJEMPLO DE PREGUNTAS DIRIGIDAS A RELACIONAR UNAS IDEAS CON OTRAS.}

"A veces, los superpetroleros causan daños en la vida marina y en las costas. Especifica qué tipo de daños producen".

\section{EJEMPLO DE PREGUNTAS DE APLICACION DE LA INFORMACION DEL TEXTO A SITUACIONES NUEVAS.}

"Hay grandes camiones que transportan mercancías peligrosas. A veces, estos camiones tienen accidentes que ocasionan graves daños. Fijándote en la información del texto, ¿qué consejos darías para evitar o disminuir los accidentes de esos camiones y los daños que causan? Explica las medidas que se te ocurran, comentando en cada caso cómo podrian ayudar esas medidas".

También hay datos cualitativos más difíciles de valorar. Así, los profesores nos comunicaron que bastantes niño y niñas decían que estaban aprendiendo a estudiar mejor, así como que comprendían más y mejor que antes de emplear la técnica. 
Comentarios sobre la utilización de la técnica de mapa de ideas

A partir de los resultados mencionados, hay argumentos para concluir que la técnica de mapa de ideas ayuda a los estudiantes a realizar esas actividades mentales que son esenciales para comprender un texto: distinguir lo esencial de lo accesorio, encontrar relaciones entre las ideas del texto, especialmente entre las más importantes, y finalmente dar una organización jerárquica a las ideas y relaciones encontradas. Nuestros resultados son congruentes con los de otros autores que emplean técnicas similares (Pearson y Fielding, 1991) y con lo que se conoce actualmente sobre el funcionamiento de la memoria humana y sobre los procesos de comprensión. Es decir, recordamos más y mejor cuando somos capaces de organizar la información. Por otra parte, comprender es, en gran medida, seleccionar, relacionar y jerarquizar información. Además, dado que esas actividades mentales se han de manifestar externamente en acciones de construcción de un mapa de ideas, y dado que se promueve la participación activa de los chicos (p.e. dando razones de sus respuestas y contrastándolas entre ellos), los muchachos van aprendiendo estrategias para regular sus propios procesos de comprensión.

Junto a estos comentarios positivos, quisiéramos añadir dos más encaminados a señalar algunas limitaciones de la técnica de mapas de ideas. En primer lugar, la técnica que hemos explicado parece que tiene efectos positivos en los procesos mentales que hemos comentado, pero no es suficiente si lo que se pretende es poner en marcha procesos que supongan la aplicación de los conocimientos que han adquirido a situaciones nuevas, tal como acabamos de mencionar. En estos casos parece que es preciso enseñar a los niños a activar sus conocimientos previos con el fin de conectarlos con las informaciones textuales, e incluir otras actividades más específicas. En segundo lugar, si los textos son poco coherentes, tienen una mala organización y presuponen una considerable cantidad de conocimiento previo por parte del estudiante, es realmente muy difícil y casi imposible que los alumnos sean capaces de realizar las actividades necesarias para construir un mapa de ideas. Desgraciadamente, es relativamente frecuente encontrar textos escolares con las carencias que acabamos de señalar. En estos casos los estudiantes no pueden seleccionar la información importante, ni pueden relacionar unas ideas con otras, ni organizarlas, ni jerarquizarlas. La explicación psicológica es clara. La comprensión se facilita si, o bien el texto, o bien el lector imponen una organización a la información (Just y Carpenter, 1987). Cuando los textos están mal organizados y son poco coherentes, y además los lectores son estudiantes que por definición tienen poco conocimiento de aquello que estudian, el texto se convierte en algo difícilmente inteligible (Vidal-Abarca, Sanjosé y Solaz, 1993). En estos casos, los estudiantes frecuentemente memorizan sin comprender realmente lo que leen, lo cual hace practicamente imposible la tarea de construir un mapa de ideas.

Para terminar, quisiéramos formular unas anotaciones adicionales. En primer lugar, creemos que son los profesores de las materias que emplean textos expositivos (Ciencias Sociales, Ciencias Fisico-Naturales) los más indicados para utilizar la técnica de mapas de ideas en sus aulas. Es decir, es importante enseñar técnicas de comprensión de textos allí donde se dan los problemas de comprensión. En segundo lugar, creemos que no se trata de hacer un mapa de ideas de cada lección, sino de enseñar la técnica a los chicos y practicarla como una herramienta más del aprendizaje de contenidos de una materia. Es decir, se trata de incorporar la actividad de construcción de mapas de ideas al proceso ordinario de enseñanza, como una actividad más, utilizable cuando parezca oportuno. Además, hay que tener en cuenta que estas técnicas, como las habilidades de comprensión, no se aprenden de una vez para siempre, sino que suponen un largo proceso de aprendizaje que hay que aplicar flexiblemente a distintos tipos de textos (mejor y peor elaborados) pertenecientes a diversas materias con características distintas (p.e. la Física es bien diferente de la Geografía) y sobre las que los estudiantes tienen distinto grado de conocimiento previo. En este 
sentido, compartimos el planteamiento general de Sánchez (1993): insertar actividades de comprensión en el currículum ordinario, siendo cuidadosos y rigurosos a la hora de decidir qué actividades se incorporan, cuándo y cómo se debe hacer dicha incorporación. En tercer y último lugar, quisiêramos resaltar que la técnica de los mapas de ideas puede ser positiva en la medida en que hace que los alumnos realicen actividades mentales que facilitan la adquisición de estrategias de comprensión de textos. Ciertamente no es la única técnica para conseguir este objetivo. Se trata simplemente de una herramienta que el profesor puede utilizar como un elemento más de la enseñanza, conociendo tanto sus posibilidades como sus limitaciones.

\section{Referencias}

ARMBRUSTER, B. B., y ANDERSON, T. H. (1982). Idea-mapping: the technique and its use in the classroom or simulating the "ups» and "downs" of reading comprehension. Tech. Rep. $\mathrm{n}^{\circ} 36$. Center for the Study of Reading. University of Illinois at Urbana-Champaign.

Fernandez, T. (1992). Mapas conceptuales y diagramas UVE: dos estrategias en la enseñanza de la historia. Comunicación, Lenguaje y Educación, 16, 7-24.

Geva, E. (1985), Mejora de la comprensión lectora mediante diagramas de flujo. Infancia y Aprendizaje, $31-32,45-66$.

Holley, C. D., y Dansereau, D. F. (1984). Networking: The technique and the empirical evidence. En C.H. Holley \& D.F. Danserau (Eds.), Spatial Leaming Strategies. Techniques, applications, and related issues (pp. 81-108). Nueva York: Academic Press.

JUST, M. A., y CARPENTER, P. A. (1987). The psbycbology of reading and language comprebension. Boston: Allyn \& Bacon.

NovaK, J. D., y GowIN, D. B. (1988). Aprendiendo a aprender. Barcelona: Martínez Roca.

Pearson, P. B., y Fielding, L. (1991). Comprehension instruction. En R. Barr, M.L. Kamil, P.B. Mosenthal y P.D. Pearson, (Eds.), Handbook of reading Research, Vol II (pp. 815-860). Nueva York: Longman.

SANCHEZ, E. (1993). Los textos expositivos. Estrategias para mejorar su comprensión. Madrid: Santillana.

VIDAL-ABARCA, E., y GILABERT, R. (1991). Comprender para aprender. Madrid: Cepe.

VIDAl-AbarCA, E.; GILABERT, R., y Garcia MAdruga, J. A. (1993) (en prensa). Idea-mapping techniques: effects on recall, comprehension and learning. En B. van HoutWolters and F. de Jong (Eds.), Process-oriented instruction. Verbal and pictorial aid and comprebension strategies. Amsterdam: VU University Press.

VidAL-ABARCA, E.; SANJOSE, V., y SOlAZ, J. J. (1993). Efectos de las adaptaciones textuales, el conocimiento previo y las estrategias de estudio en el recuerdo, la comprensión y el arendizaje de textos científicos. Infancia y Aprendizaje (aceptado y pendiente de publicación). 


\section{Mapas de ideas: una herramienta para el aprendizaje escolar. Datos y comentarios para una discusión Eduardo Vidal-Abarca y Ramiro Gilabert $C L \& E, 1994,21, p p .75-86$}

Resumen: El presente trabajo hace una revisión de la técnica de «mapa de ideas», una técnica para mejorar la comprensión y aprendizaje de textos de los alumnos, y describe la utilización que los autores han hecho de la misma. Comenzamos exponiendo los fundamentos psicológicos de dicha técnica con el fin de ofrecer una base sólida a la propuesta didáctica. A continuación hacemos una revisión de la técnica y describimos de forma minuciosa el procedimiento de intervención seguido por nosotros en un programa llevado a cabo con chicos de $6^{\circ}$ de E.G.B., incluyendo un breve resumen de los principales resultados. Finalmente, hacemos unos comentarios sobre las posibilidades y limitaciones que tiene la técnica «mapa de ideas» como herramienta didáctica para mejorar la comprensión y el aprendizaje de los alumnos.

Dirección: Departamento de Psicología Evolutiva y de la Educación. E. U. de Formación del Profesorado de E.G.B. Universidad de Valencia. E. U. de Formación del Profesorado de E.G.B. C/ Alcalde Reig, 8. 46006 Valencia. Teléfono: 93-38644 80, Ext. 7266.

Datos de los autores: Los autores de este trabajo son profesores del Departamento de Psicología Evolutiva y de la Educación en la Escuela Universitaria de Formación del Profesorado de E.G.B. y en la Facultad de Psicología de la Universidad de Valencia. Han trabajado como profesores de E.G.B. en diversos centros escolares durante 13 años antes de incorporarse a la universidad. Desde hace varios años están investigando en temas relacionados con la comprensión y aprendizaje de textos.

(C) PERMISOS PARA CITAR O REPRODUCIR EN OTRAS FUENTES: Se pueden citar libremente hasta 500 palabras. Para reproducir una porción de texto mayor, figuras o ilustraciones, se deberá pedir permiso por escrito a la revista, especificando el uso al que se destina el texto. En todos los casos, se deberá citar el copyright de $C L \& E$. En el caso de artículos o textos que hayan sido a su vez reproducidos en $C L \& E$ los interesados deberán dirigirse tanto a los detentadores del copyright original como a $C L \& E$, en el caso de que se quiera hacer uso de la traducción. FOTOCOPIAS: Para todo lo relacionado con el uso mediante fotocopia del material de esta revista, deberán dirigirse a: CEDRO, C/ José Marañón, $10,3 .^{\circ}$ Izda. Tel. 5941575 . Fax 4453567. 